Forum Kind Jugend Sport $2020 \cdot 1: 130-137$ https://doi.org/10.1007/s43594-020-00023-4 Online publiziert: 6 . November 2020

(c) Deutsche Sportjugend im DOSB e.V. 2020

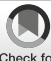

Katharina Morlang

Deutsche Sportjugend, Frankfurt am Main, Deutschland

\title{
Das Selbstverständnis von Trainer*innen
}

\section{Auszug aus der Evaluation zum Projekt "TrainerlnSportdeutschland"}

zogen. Aus den Ergebnissen können Einschätzungen ermittelt und Handlungsempfehlungen abgeleitet werden, um Trainer ${ }^{\star}$ innen in ihrer Rolle besser zu unterstützen.

\section{Die Interessen von Kindern und Jugendlichen im Sport}

Das Selbstverständnis der Trainerinnen ist ein noch wenig erforschtes Feld im Sport. Insbesondere für den Kinder- und Jugendsport ist es jedoch eine gesamtgesellschaftliche Aufgabe, zu hinterfragen, welche Rolle Trainer*innen sich selbst zuschreiben und wie sie meinen, ihr Training für Kinder und Jugendliche zu gestalten. Trainer*innen sind diejenigen Personen, mit denen Kinder und Jugendliche die meiste Zeit im organisierten Sport verbringen - die jungen Sportler*innen werden maßgeblich von ihnen geprägt. Die grundlegenden Fragestellungen in diesem Beitrag sind daher: Welches Selbstverständnis haben Trainer*innen bezüglich ihrer Tätigkeit als Trainer ${ }^{\star}$ in - und wie schätzen die Institutionen, für die sie tätig sind (hier: Sportverbände), dies im Vergleich dazu ein? Sehen Trainer*innen sich eher als Expert*innen für sportpraktische Inhalte, mit deren Hilfe die sportliche Leistung ihrer Athlet*innen gesteigert werden kann - oder fühlen sie sich als Vermittler*innen darüber hinaus liegender persönlicher Kompetenzen, also als Fördernde von Sportpersönlichkeiten? Für eine Beantwortung dieser Fragen wird der diesbezüglich relevante Teil einer Evaluation zum Projekt „TrainerInSportdeutschland“ (2020) herange-
Um der Frage nachzugehen, über welche Kompetenzen und welche Rolle Trainer*innen idealerweise verfügen sollten - also welches Selbstverständnis Trainer*innen für ein kind- und jugendgerechtes Training brauchen, um damit positive Rahmenbedingungen für sportliche und persönliche Entwicklung zu schaffen - sollte von den Interessen der Kinder und Jugendlichen ausgegangen werden. Unterschiedliche Quellen in der Fachliteratur (u. a. Schmidt et al. 2015a) stellen fest: Kinder und Jugendliche wollen vor allem Spaß am Sport haben. Die Kinder und Jugendlichen kritisieren, beispielsweise auch in der aktuellen SINUS-Jugendstudie (SinusInstitut 2020), dass häufig zu viel Leistungsdruck im Verein besteht. Sie sind allerdings nicht per se gegen Wettkämpfe. Wenn Wettkämpfe gezielt für eine positive Entwicklung eingesetzt werden, können sie viele Kinder und Jugendliche anspornen. Junge Menschen wollen sich selbstverständlich auch in ihrer Sportart verbessern und gefordert werden; laut Richartz et al. spielt Kompetenzerleben (Richartz et al. 2007, S. 33) dabei eine wichtige Rolle. Aber sie gehen auch in den Sportverein, um ihre Freund ${ }^{*}$ innen $\mathrm{zu}$ treffen und Gemeinschaft $\mathrm{zu}$ er- leben. Im Rahmen des von der EU geförderten und vom Knowledge Centre for Sport Netherlands in den Jahren 2017 bis 2019 durchgeführten Projekts „Keep youngsters involved“ wurde ermittelt, welche Rahmenbedingungen der Drop-out-Prävention dienen (https:// en.kenniscentrumsportenbewegen.nl/ international-projects/keep-youngstersinvolved/products-and-tools/):

- beteiligt werden, mitentscheiden am Training,

- sich verbessern, aber auch Freunde treffen etc.,

- gewisse Freiheiten haben, um mitentscheiden zu dürfen,

- selbst wirksam sein, Fähigkeiten erlernen, die sie weiterentwickeln können und mit denen sie Herausforderungen begegnen können,

- Beziehungen aufbauen, Gemeinschaft, Akzeptanz, Sicherheit haben,

- in einem positiven Lernklima aufwachsen und Feedback erhalten, insbesondere zum und durch den/die Trainer*in,

- flexibler Sport treiben können und dies gut mit ihrer Freizeit/Schule vereinbaren,

- Anerkennung und Wertschätzung.

Richartz et al. (ab S. 287) nennen noch weitere Merkmale von idealen Trainer*innen für Kinder. Sie wollen Begleiter*innen, die sie motivieren und mit denen man gemeinsam Spaß haben kann. Die aufgezählten Aspekte können je nach Geschlecht, Alter, Herkunft, Kultur und sozio-ökonomischem Status variieren, bleiben aber im Wesentlichen 
für alle Kinder gleich. Trainer ${ }^{*}$ innen sollten diese Bedürfnisse von Kindern und Jugendlichen kennen und die individuellen Unterschiede berücksichtigen.

Welche Kompetenzen müssen Trainer*innen mitbringen, um diesen Anforderungen gerecht $\mathrm{zu}$ werden? Von Gesellschaft und Politik und auch in den Sportverbänden selbst wird der ${ }^{\star}$ die Trainer*in häufig als Lernbegleiter ${ }^{\star}$ in, Fördernde von Persönlichkeiten und als Schlüsselfigur für die gesunde Entwicklung von Kindern und Jugendlichen betrachtet.

\section{Das Grundverständnis zur „pädagogischen Trainingsqualität ${ }^{\prime \prime}$}

Kinder treiben in erster Linie Sport, weil sie Freude daran haben. Im dritten Kinder- und Jugendsportbericht heißt es dazu: Der Spaß am Sport ist das Fundament sportlichen Engagements, das alle Trainer*innen, Funktionär*innen und Eltern berücksichtigen und weiterentwickeln sollten (Schmidt et al. 2015b, S. 556). Freude am Sport ist insbesondere dann möglich, wenn Trainer*innen über pädagogische Kompetenzen verfügen: Wenn sie die eigene Trainingsphilosophie reflektieren und ein Selbstverständnis entwickeln, um grundsätzlich an den Bedürfnissen der Kinder und Jugendlichen anzuknüpfen, damit Dropout vermieden wird, damit Kinder und Jugendliche mit Spaß und Leidenschaft beim Sport bleiben und damit sie sowohl ihre persönlichen als auch motorischen Kompetenzen weiterentwickeln können. Dieser „pädagogischen Trainingsqualität“ liegt ein ganzheitlicher Bildungsansatz zugrunde, der auf die Förderung von Kompetenzen mit vielfältigen $\mathrm{Me}$ thoden abzielt. Freiwilligkeit, Partizipation und die Orientierung an den Bedürfnissen und Interessen der jungen Sportler*innen sind dabei maßgeblich. Ziel ist es, Kinder und Jugendliche in den Mittelpunkt des Sporttreibens zu stellen und von ihren Bedürfnissen und Potenzialen ausgehend Training zu gestalten. Idealerweise bildet ein Grundverständnis von pädagogischer Trainingsqualität die Grundlage für alle Angebote des Kinder- und Jugendsports und wendet sich insbesondere an die Verantwortlichen in den Qualifizierungen von Trainer ${ }^{\star}$ innen, die Sportvereine und -verbände sowie direkt an alle Trainer*innen.

Trainer ${ }^{\star}$ innen haben somit die Aufgabe - entsprechend den beschriebenen Bedürfnissen junger Menschen -, ihnen Freude am Sport zu vermitteln und ihre Persönlichkeit im Sport soweit zu fördern, dass sie eigenverantwortlich Entscheidungen treffen und langfristig sportlich aktiv bleiben - in allen Bereichen, ob Breiten-, Leistungs- oder Spitzensport.

Darüber hinaus haben Kinder und Jugendliche ein Recht auf Bildung, Schutz, Beteiligung, Gesundheit, Bewegung und eine eigene Stimme (UN-Kinderrechtskonvention o.J.). Sportverbände und -vereine sollten diese Rechte von Kindern und Jugendlichen zusammen mit ihren Trainer*innen wahren und vermitteln. In den Rahmenrichtlinien des Deutschen Olympischen Sportbundes (DOSB o.J., S. 14-17 und S. $43 \mathrm{ff}$.) wird zwar die Vermittlung sozialer und pädagogischer Kompetenzen von Trainer*innen gefordert, in den Ausbildungen findet das aber noch nicht durchgängig Berücksichtigung.

\section{Datengrundlage und Ziele der Evaluation}

Um zu prüfen, welches Selbstverständnis Trainer*innen haben und inwiefern ihnen die Reichweite ihrer Aufgaben und Verantwortung bewusst ist, bildet eine interne Evaluation im Rahmen des Projekts „TrainerInSportdeutschland“, die im April 2020 mit den ersten Projektteilnehmenden durchgeführt wurde, die Datengrundlage. Ziel des Projekts ist die Verbesserung der Situation der Trainer*innen in ganz Deutschland, um der Vision, bis 2026 ausreichend qualifizierte Trainer*innen in Sportdeutschland zu gewinnen, näherzukommen (https:// trainerinsportdeutschland.dosb.de/). Zu Beginn der ersten Projektphase wurde eine Evaluation der 16 beteiligten Trainer*in-Projekte, also sowohl der jeweiligen Verbände als auch der beteiligten Trainer*innen durchgeführt. Ziel war es zu überprüfen, wie sich der Ist-Zustand bezüglich der Leitziele der Vision (https://trainerinsportdeutschland.dosb. de/vison/) von „TrainerInSportdeutschland" darstellt. Unter anderem wurde ermittelt, ob die Arbeitsbedingungen für Trainer*innen attraktiv sind, ob ein Berufsbild existiert, ob Trainer*innen sich wertgeschätzt und anerkannt fühlen, ob sie als Kernaufgabe im Verband verstanden werden und im Rahmen einer systematischen Personalentwicklung gefördert werden, ob sie sich partizipativ einbringen dürfen und vieles mehr. Darüber hinaus wurde in einem kleinen Teil der Evaluation nach dem Selbstverständnis der Trainer*innen gefragt, also inwiefern sie die Persönlichkeit der Sportler*innen fördern und sich selbst als Lernbegleiter ${ }^{\star}$ innen verstehen. Die Fragebögen wurden mit einer LikertSkala zur Messung persönlicher Einstellungen und freien Textfeldern versehen. Die Evaluation ist nicht repräsentativ für alle Trainer*innen in Deutschland, erlaubt allerdings einen Einblick in das Selbstverständnis des Berufs und gibt Hinweise für weitere Forschungsfragen.

\section{Trainer*innen als Lern- begleiter*innen}

Die Evaluation gibt zunächst Aufschluss über das Profil der beteiligten Trainer*innen im Projekt „TrainerInSportdeutschland“. Befragt wurden 176 Trainer*innen aus 16 Trainer ${ }^{*}$ in-Projekten. Das Durchschnittsalter beträgt 43,6 Jahre, vorwiegend sind sie männlich $(68 \%$ männlich, $32 \%$ weiblich). $60 \%$ der Trainer*innen sind im Verein, $18 \%$ auf Bundesebene und $24 \%$ auf Landesebene aktiv (da Trainer*innen auf unterschiedlichen Ebenen aktiv sein können, ist die Summe hier größer als $100 \%)$. Im Durchschnitt sind Trainer*innen befragt worden, die stark an der Vereinsbasis tätig sind. Genau zu je $43 \%$ ist die eine Hälfte hauptberuflich und die andere Hälfte ehrenamtlich tätig. Nur $14 \%$ üben die Trainer*intätigkeit nebenberuflich aus. Es ist von einer bildungsnahen Gruppe auszugehen, 91\% haben eine aktuell gültige (DOSB)-Lizenz und 30\% ein sportwissenschaftliches Studium. Mehr als die Hälfte $(66,5 \%)$ trainiert hauptsächlich Leistungssportler*innen, $21 \%$ ambitionierte Freizeitsportler ${ }^{\star}$ innen und 

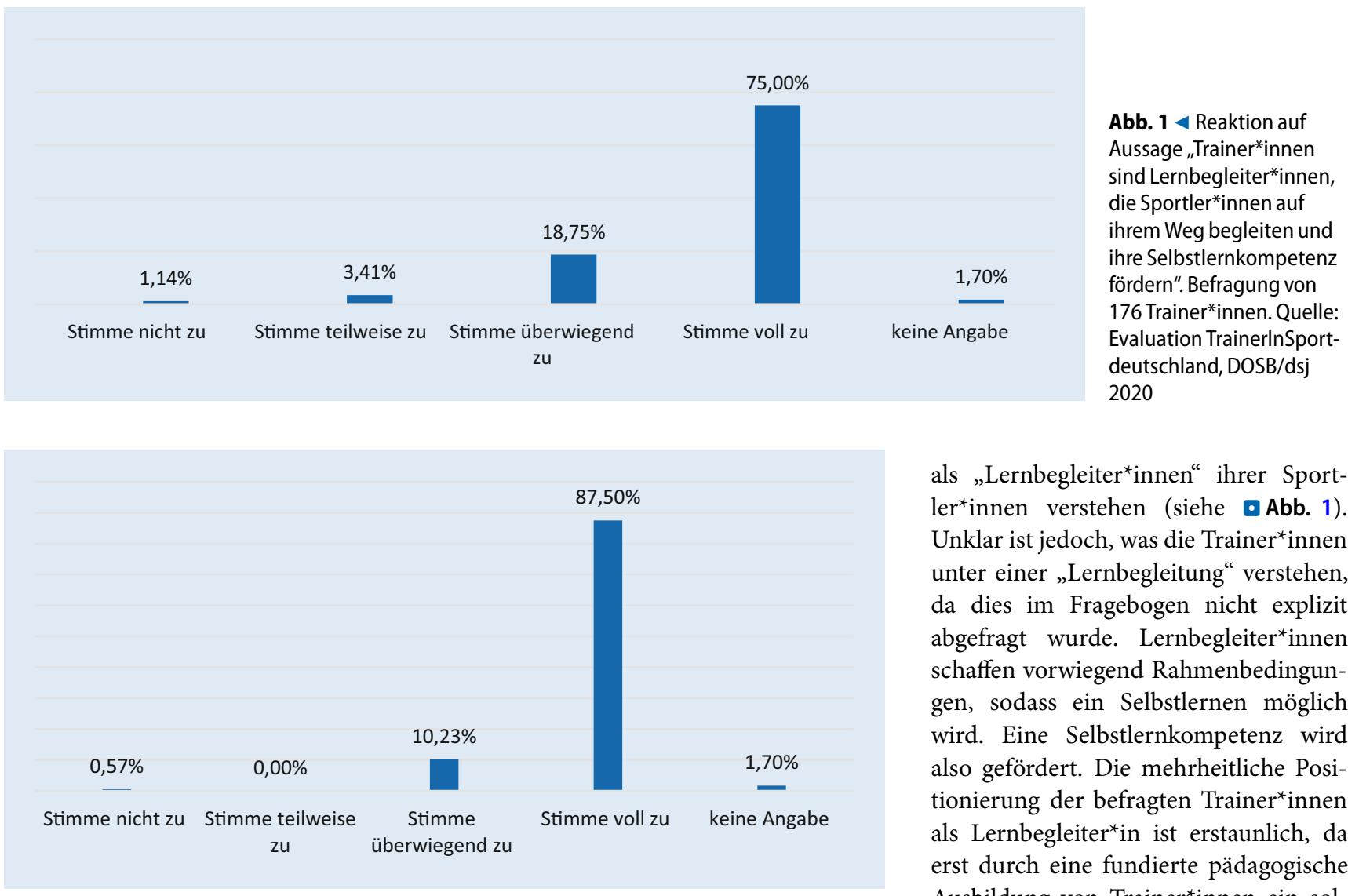

Abb. 2 ム Reaktion auf Aussage "Mir ist die Persönlichkeitsentwicklung der Sportler*innen wichtig“. Befragung von 176 Trainer*innen. Quelle: Evaluation TrainerInSportdeutschland, DOSB/dsj 2020

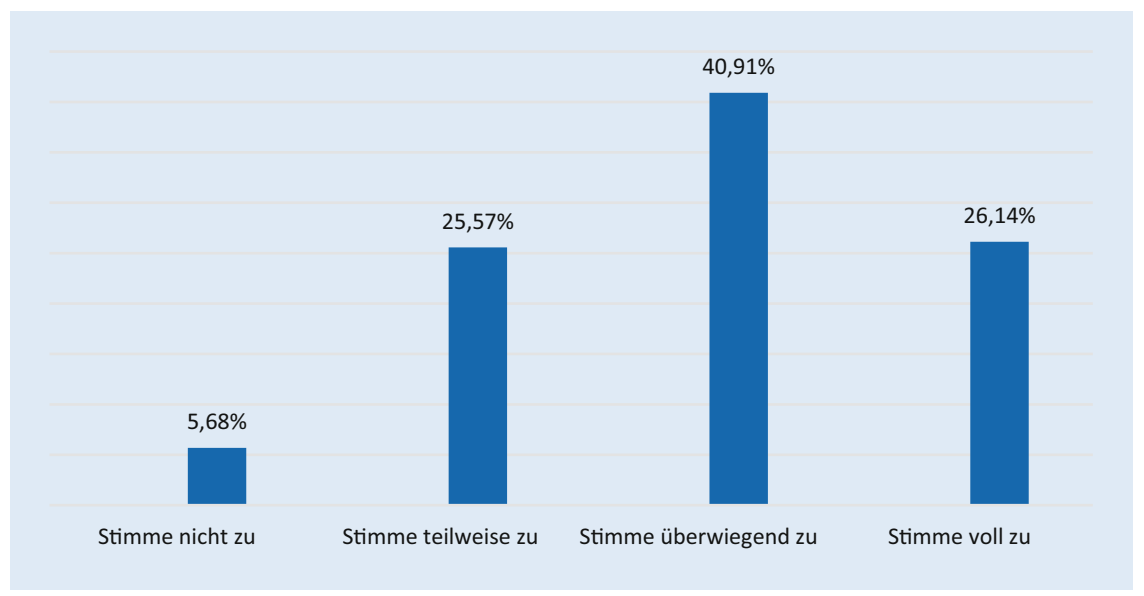

Abb. 3 A Reaktion auf Aussage „Die Leistung der Sportler*innen steht im Vordergrund meiner Tätigkeit". Befragung von 176 Trainer*innen. Quelle: Evaluation TrainerInSportdeutschland, DOSB/dsj 2020

$22 \%$ Breitensportler*innen. Aus der Zielgruppenabfrage ist ersichtlich, dass es sich vorwiegend um Jugend- beziehungsweise Kindertrainer*innen handelt: $82 \%$ trainieren Jugendliche, $56 \%$ Kinder, $44 \%$ Erwachsene und $7 \%$ Senior ${ }^{*}$ innen. Es handelt sich bei dieser Stichpobe vorwiegend um männliche Jugendtrainer*innen im Leistungssport, mit einem Durchschnittsalter von 43 Jahren und einer gültigen DOSB-Lizenz, die zum großen Teil an der Basis tätig sind.

Die Mehrheit dieser Trainer*innen (75\%) gibt an, dass sie sich selbst als „Lernbegleiter*innen“ ihrer Sportler*innen verstehen (siehe $\bullet$ Abb. 1). Unklar ist jedoch, was die Trainer*innen unter einer "Lernbegleitung" verstehen, da dies im Fragebogen nicht explizit abgefragt wurde. Lernbegleiter ${ }^{*}$ innen schaffen vorwiegend Rahmenbedingungen, sodass ein Selbstlernen möglich wird. Eine Selbstlernkompetenz wird also gefördert. Die mehrheitliche Positionierung der befragten Trainer*innen als Lernbegleiter ${ }^{\star}$ in ist erstaunlich, da erst durch eine fundierte pädagogische Ausbildung von Trainer*innen ein solches Selbstverständnis generiert werden kann - dies ist bei der Mehrheit der Befragten nicht der Fall. Sollten die Trainer*innen hier keine sozial erwünschte Antwort gegeben haben, so wäre es durchaus interessant zu erfahren, in welchem Teil ihrer Ausbildung oder ihrer Erfahrung in der Sportpraxis sie zu dieser Erkenntnis gelangt sind.

$87,5 \%$ der Befragten ist die Persönlichkeitsentwicklung der Sportler*innen wichtig (siehe $\bullet$ Abb. 2). Es scheint somit ein großes Bewusstsein für die ganzheitliche Entwicklung der Sportler*innen zu geben. Diesen Eindruck bekräftigt die Feststellung, dass zwar für $67 \%$ der Trainer*innen die Leistung der Sportler*innen im Vordergrund der Tätigkeit steht, allerdings $30 \%$ dem nur teilweise oder gar nicht zustimmen (siehe - Abb. 3). Daraus wird deutlich, dass einige Trainer*innen nicht nur die Leistung der Sportler*innen im Blick haben und sich als Ausbilder*innen verstehen, die motorische und sportartspezifische Kompetenzen von Sportler*innen fördern, sondern dass sie andere Werte und 


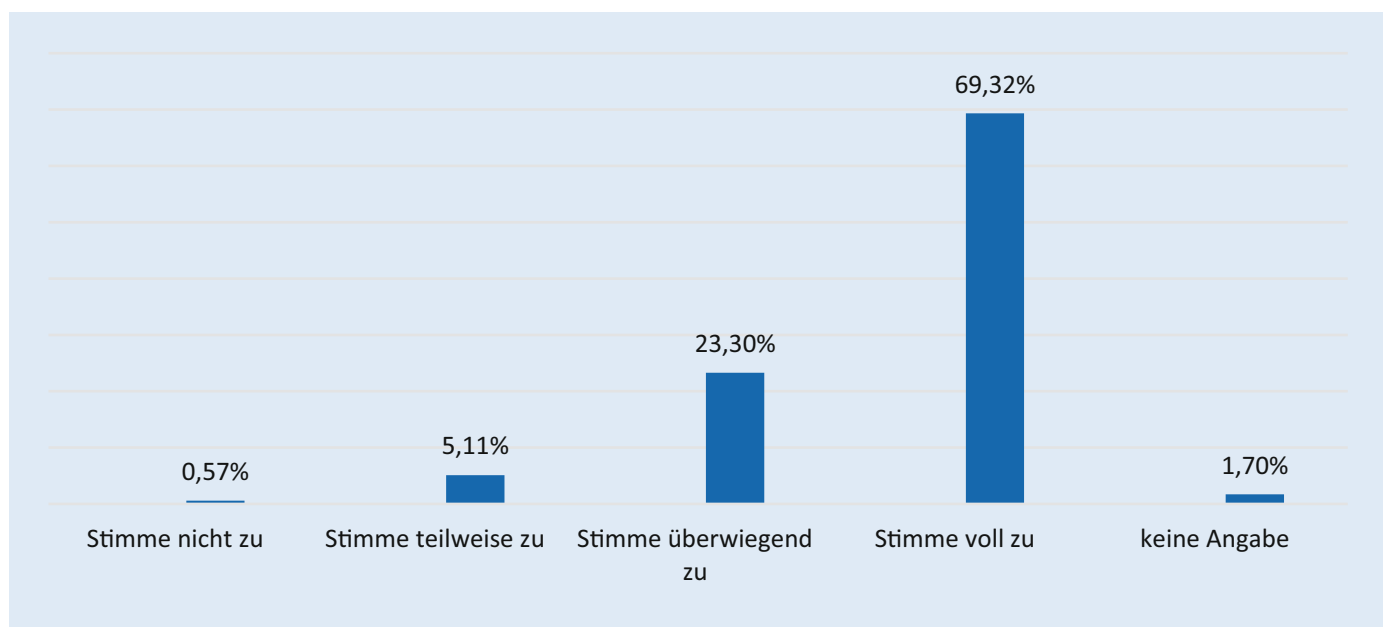

Abb. $4<$ Reaktion auf Aus sage "Ich verstehe mich als Ausbilder*in, die/der persönliche Kompetenzen von Sportler*innen fördert". Befragung von 176 Trainer*innen. Quelle: Evaluation TrainerInSportdeutschland, DOSB/dsj 2020

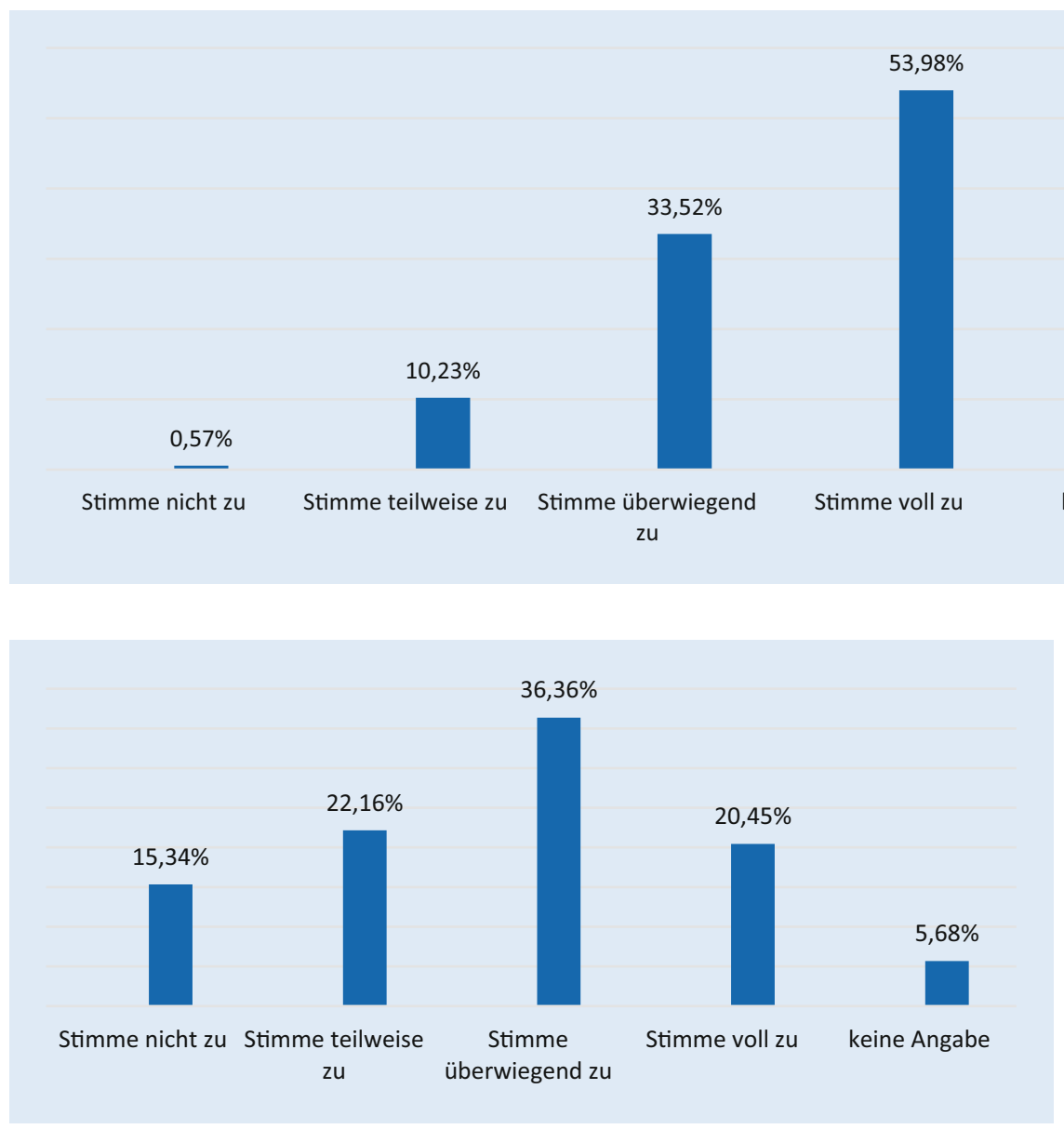

Abb. 6 A Reaktion auf Aussage „Ich entwickele gemeinsam mit den Sportler*innen ihren Trainings-

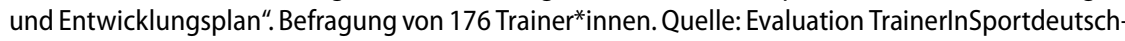
land, DOSB/dsj 2020

Ziele für sich definieren, die Vorrang haben.

Spannend wird es, wenn es um die Umsetzung des eigenen Selbstverständnisses geht (siehe • Abb. 4). Hier sind es nur noch $69 \%$ der Trainer ${ }^{*}$ innen, die voll zustimmen, dass sie die persönlichen Kompetenzen von Sportler*innen in der Trainingspraxis fördern. $93 \%$ der Trainer ${ }^{*}$ innen versuchen selbst Vorbilder für junge Sportler*innen zu sein und sie trotz unterschiedlicher Voraussetzun- gen am Sport zu beteiligen. Was einigen eher schwer fällt, obwohl diese Zahl immer noch durchschnittlich hoch ist (54\% stimmen voll $\mathrm{zu}, 34 \%$ stimmen überwiegend $\mathrm{zu}$ ), ist die Orientierung an den Potenzialen der Sportler*innen und die individuelle Förderung (siehe - Abb.5). Hier scheint es eine gewisse Unsicherheit zu geben. Ein ebenso uneinheitliches Bild zeigt sich bei der Einbindung der Sportler*innen bei der gemeinsamen Entwicklung von Trainingsplänen (siehe Abb. 6). Hier setzen sich immerhin knapp 57\% der Trainer*innen regelmäBig damit auseinander, während $43 \%$ sich zurückhaltender zeigen, Sportlerinnen hierbei aktiv einzubinden beziehungsweise keine Angabe machen. Die Mehrheit der Trainer*innen inszeniert bewusst Lernprozesse, allerdings zeigt sich auch hier eine Ambivalenz zu ihrem Selbstverständnis: Der Aussage wird deutlich seltener zugestimmt (66\%), als es das Selbst- 


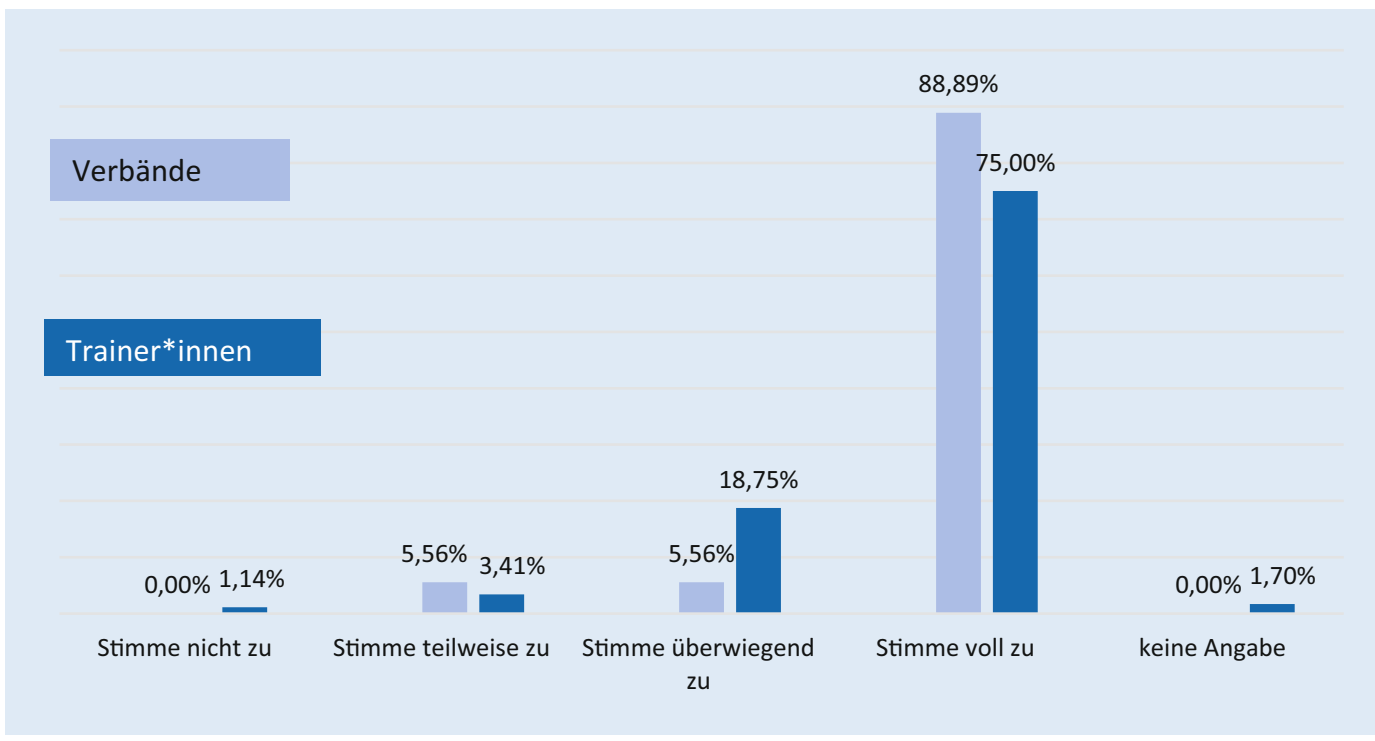

Abb. $7 \triangleleft$ Reaktion auf Aussage „Trainer*innen sind Lernbegleiter*innen die die Selbstlernkompetenz ihrer Sportler*innen fördern". Befragung von 176 Trainer*innen und 16Sportverbänden. Quelle: Evaluation TrainerInSportdeutschland, DOSB/dsj 2020

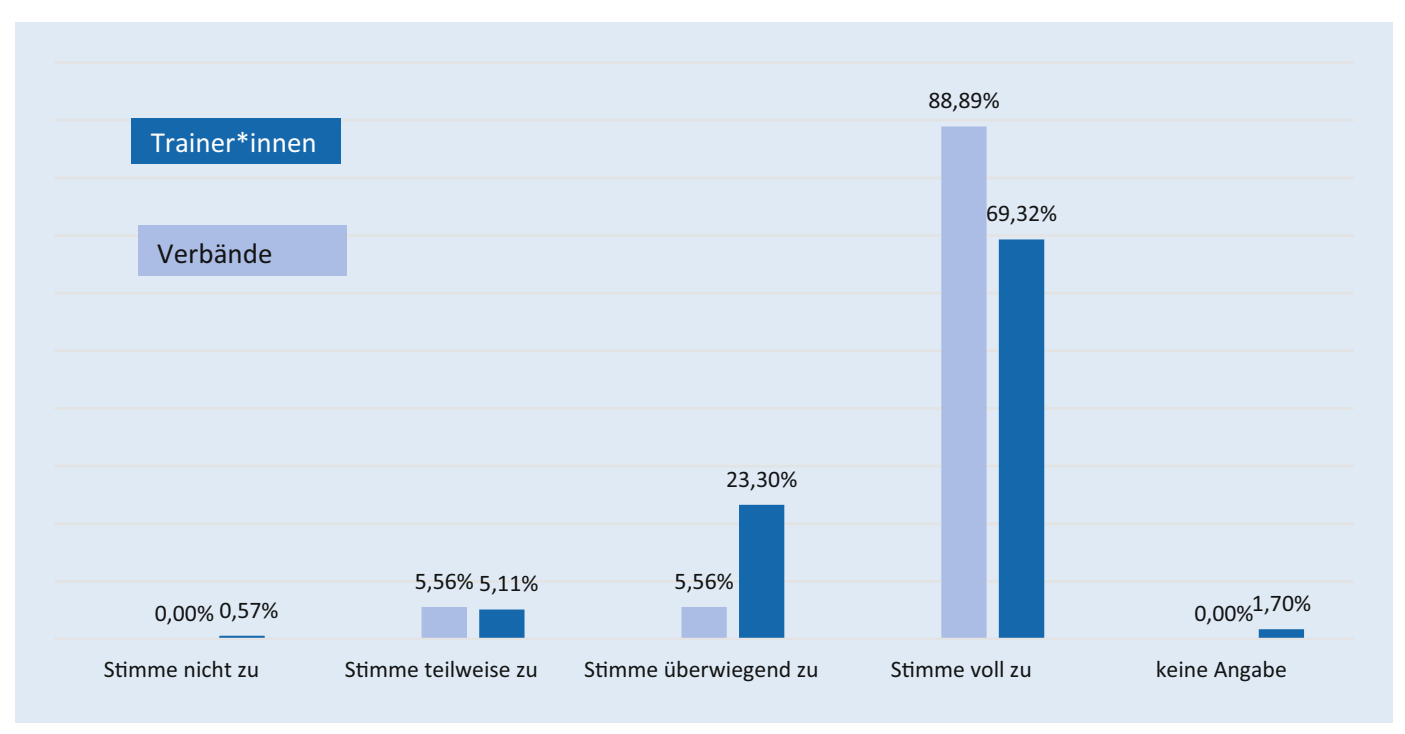

Abb. $8<$ Reaktion auf Aussage "Trainer*innen sind Ausbilder*innen, die persönliche Kompetenzen von Sportler*innen fördern". Befragung von 176 Trainer*innen und 16 Sportverbänden. Quelle: Evaluation TrainerInSportdeutschland, DOSB/dsj 2020

verständnis vermuten ließe. Die Befunde legen den Schluss nahe, dass die Trainer*innen ein umfassendes Verständnis ihrer Trainer*innenrolle haben und, ganz allgemein betrachtet, ihnen die Persönlichkeitsentwicklung von jungen Sportler ${ }^{\star}$ innen sehr am Herzen liegt. Ihnen ist bewusst, welche Aufgabe und Verantwortung sie haben. Betrachtet man allerdings konkrete Methoden, also die Umsetzung in der Praxis, so ist festzustellen, dass bei noch einigen Trainer ${ }^{*}$ innen Unsicherheiten herrschen. Die Berücksichtigung individueller Potenziale, die bewusste Steuerung von Lernprozessen und die partizipative Einbindung von Sportler*innen scheinen noch nicht flächendeckend zu gelingen.

\section{Die Rolle der Trainer*innen aus Sicht der Verbände}

Bei der internen Evaluation zum Projekt „TrainerInSportdeutschland“ wurden auch die Verbände der 16 beteiligten Trainer*in-Projekte befragt. Zur Wahrung der Anonymität soll hier lediglich der Hinweis erfolgen, dass die Verbände größere Landes- und Spitzenverbände sind. Die Ergebnisse zeigen, dass 89\% der Verbände dem zustimmen, dass Trainer*innen ihre Sportler*innen in ihren Lernprozessen begleiten und ihre Fähigkeiten, sich eigenverantwortlich und persönlich zu entwickeln, fördern sollten (siehe - Abb. 7 und 8). Mehr Zustimmung (94\%) erfährt der Standpunkt, dass Trainer*innen für die motorischen und sportartspezifischen Kompetenzen von Sportler*innen zuständig sind (siehe - Abb.9). Dies steht für alle Verbände außer Frage. Hinsichtlich der beiden Kernfragen sind keine relevanten Unterschiede zu sehen, damit ist davon auszugehen, dass Verbände ihren Trainer*innen eine wichtige Rolle als Lernbegleiter ${ }^{\star}$ innen von Sportler*innen zuschreiben, die ihre persönlichen Kompetenzen fördern, und gleichzeitig eine sportartspezifische Ausbildung der Sportler*innen im Vordergrund steht. Auffallend ist, dass die befragten Verbände ihre Mitgliedsorganisationen nur selten für das Thema der Lernbegleitung sensibilisieren. Die Verbände wissen 


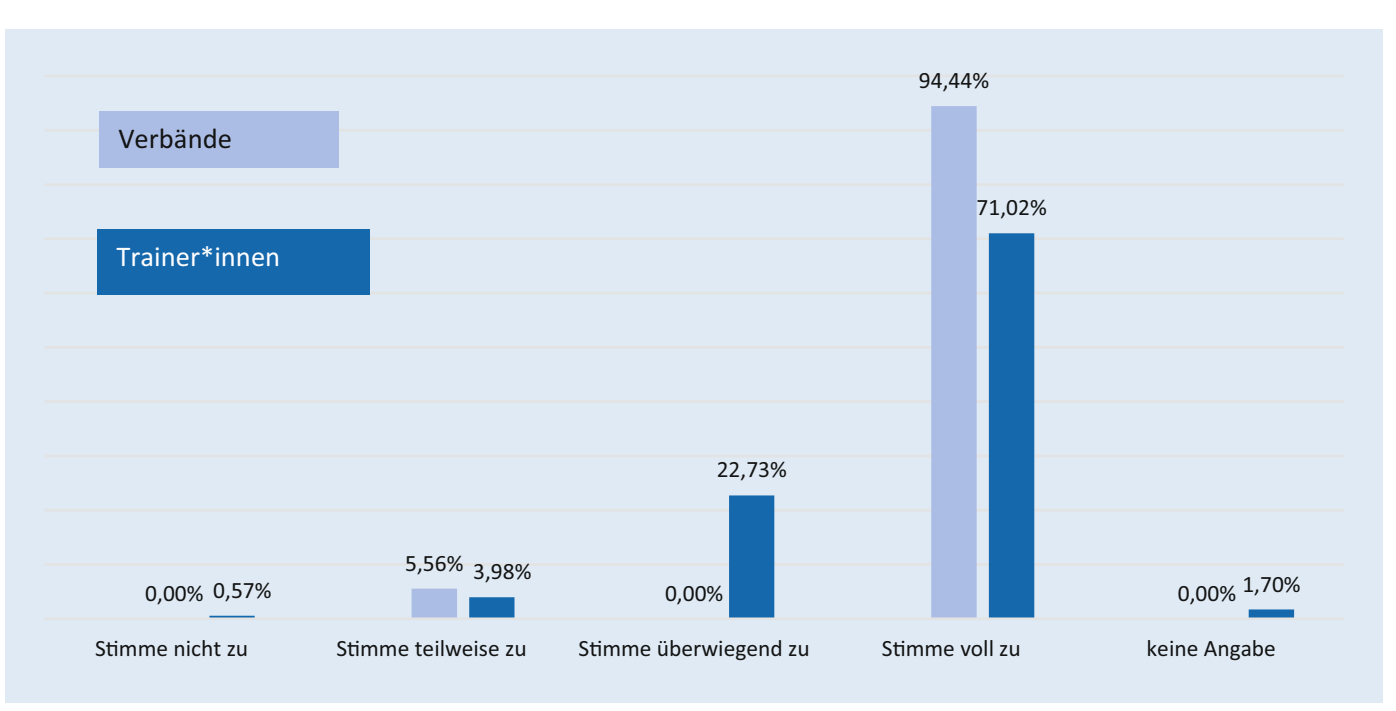

Abb. $9 \triangleleft$ Reaktion auf Aussage „Trainer*innen sind Ausbilder*innen, die motorische und sportartspezifische Kompetenzen von Sportler*innen fördern". Befragung von 176 Trainer*innen und 16 Sportverbänden. Quelle: Evaluation TrainerInSportdeutschland, DOSB/dsj 2020

zwar um die Wichtigkeit der Rolle der Trainer*innen für die Förderung der persönlichen und motorischen Kompetenzen von Sportler*innen, aber sie scheinen dieses Bewusstsein nicht in die Strukturen des Sports weiterzureichen.

\section{Die zentralen Erkenntnisse}

Aus den geschilderten Ergebnissen lassen sich zentrale Erkenntnisse ableiten: In den befragten Verbänden nehmen noch immer wesentlich mehr Männer als Frauen eine Tätigkeit als Trainer*in auf, was die Förderung von Frauen grundsätzlich weiter in den Blick rückt. Die meisten Trainer*innen sind auf der Vereinsebene tätig und verfügen über eine gültige DOSB-Lizenz. Die Trainer*innen sind hauptsächlich im Leistungssport tätig und trainieren größtenteils Kinder und Jugendliche.

Es ist zu berücksichtigen, dass Trainer*innen und Verbände sozial erwünschte Antworten gegeben haben könnten. Daher sind die Erkenntnisse als subjektive Vermutungen anzusehen. Einige Trainer ${ }^{\star}$ innen stellen die Leistung eher hinten an, in ihrem Training hat die Persönlichkeitsentwicklung Priorität. Bei der konkreten Umsetzung ist allerdings fraglich, ob sie auch über ausreichendes Know-how verfügen, um persönliche Kompetenzen bei Sportler*innen zu fördern. Auch der Verband positioniert sich klar zur Rolle der Trainer*innen: Sie sollen motorische, aber auch persönliche Fähigkeiten der Sport- ler*innen ausbauen. Allerdings geben die Verbände diese Erkenntnisse eher selten an ihre Untergliederungen weiter.

Trainer*innen berücksichtigen, obwohl ihnen die persönliche Entwicklung junger Sportler*innen wichtig ist, seltener individuelle Potenziale und binden Sportler*innen auch seltener in partizipative Entscheidungen ein. Es liegt die Vermutung nahe, dass den befragten Trainer*innen nicht deutlich ist, welche Aspekte einer Lernbegleitung und der Persönlichkeitsentwicklung von Menschen zugrunde liegen. Welche konkreten Ansatzpunkte in ein Training einfließen müssen und was dabei zu berücksichtigen ist, scheint vielen Trainer*innen nicht bewusst, obwohl sie sich selbst in der Rolle als Lernbegleiter ${ }^{\star}$ in sehen. Bei den Verbänden könnte wiederum die Vermutung naheliegen, dass sie die Stärkung des Selbstverständnisses von Trainer*innen bisher eher selten in Angriff genommen haben. Dabei wäre es wichtig, sowohl die Mitgliedsorganisationen dafür $\mathrm{zu}$ sensibilisieren als auch die Trainer*innen fortzubilden beziehungsweise ihnen Werkzeug an die Hand zu geben.

\section{Handlungsempfehlungen}

Die in der Evaluation befragten Trainer*innen sind sich grundsätzlich ihrer Verantwortung im Training mit Kindern und Jugendlichen bewusst. Sie wissen, welche Verhaltensmuster und Trainingsziele erwünscht und gefragt sind. Setzen sie diese in ihrer Handlungspraxis um, ist das Training kind- und jugendgerecht und trägt zu mehr Freude am Training sowie wahrscheinlich auch seltener zu einem Austritt bei. Wie aber können die Sportverbände ihre Trainer ${ }^{\star}$ innen besser im Hinblick auf ihre Rolle und Tätigkeit unterstützen? Wie können sie die Ziele, die sie definitiv gemeinsam definieren, in die Praxis umsetzen? Dazu fehlen in gewisser Hinsicht Trainingsmethoden, Instrumente der Trainingsgestaltung, Leitfäden und vielleicht auch die Möglichkeit, die eigene Trainingsgestaltung und -philosophie zu reflektieren und weiterzuentwickeln. Pädagogische Handlungskompetenz im Training ist ein wichtiges Merkmal der Trainer*inbildung, das es gilt, stärker im Blick zu haben und zu fördern.

Gleichwohl gibt es bereits zwei Modelle zur Entwicklung einer pädagogischen Trainingsqualität, die in der Trainer*inbildung Anwendung finden können. Verstehen sich Trainer*innen als Ausbilder*innen von Sportpersönlichkeiten, ist das Rahmenkonzept zur Persönlichkeits- und Teamentwicklung im Sport heranzuziehen, das 2007 von Prof. Dr. Ralf Sygusch gemeinsam mit der Deutschen Sportjugend entwickelt wurde (Sygusch und Weller 2005). Das Konzept versteht sich als Beitrag zur Entwicklung der sportlichen Handlungs- und Leistungsfähigkeit sowie zur allgemeinen Persönlichkeitsentwicklung von Kindern und Jugendlichen. Es definiert Kernziele und Methoden zur 
systematischen Förderung der ausgewählten Ressourcen Selbstwirksamkeit und Selbstkonzept, soziale Kompetenzen, sozialer Rückhalt und Gruppenzusammenhalt. Ziel ist es, Kinder und Jugendliche durch die Stärkung des Selbstkonzepts und die Ausbildung von sozialen Kompetenzen bei der Bewältigung von Alltagsanforderungen zu unterstützen, sie gleichermaßen an den Sport zu binden und in ihrer Leistungsentwicklung zu fördern. Dabei wird der Bogen zwischen der Jugendarbeit im Sport im Interesse der ganzheitlichen Entwicklung von Kindern und Jugendlichen als auch zwischen der Nachwuchsund Leistungsförderung im Interesse des Sports gespannt.

Für die geplante Förderung der Persönlichkeits- und Teamentwicklung sollten Trainer*innen folgende Grundhaltung einnehmen:

Trainer ${ }^{\star}$ innen

- sind Vorbilder und sportliche Begleiter*innen,

- schaffen ein angstfreies, soziales Wohlbefinden und positives Lernklima in der Trainings- und Wettkampfgruppe,

- gehen von einem integrierten Sportverständnis aus, in dem sowohl motorische als auch psychosoziale Aspekte ihren Platz haben,

- schaffen Gelegenheiten zur Mitverantwortung und beziehen Sportler*innen in organisatorische, soziale und inhaltliche Entscheidungen ein,

- üben einen kalkulierten Leistungsdruck aus, der dem Prinzip folgt: „Fördern und fordern - nicht überfordern!“ (vgl. Sygusch 2007).

Ein weiteres Modell zur Entwicklung einer pädagogischen Trainingsqualität, das in der Trainer ${ }^{*}$ inbildung Anwendung finden könnte, bieten die Materialien, die im Rahmen des EU-Projekts ,iCoachKids"im Jahr 2018 veröffentlicht wurden. Die Federführung des Projekts liegt bei der Universität Leeds, England, das Gesamtprojekt konzentriert sich auf die pädagogische Handlungskompetenz der Trainer*innen, die förderliche Rahmenbedingungen für ein kindgerechtes Training schaffen. In drei MOOCs (Massive Open Online Courses - Online-Selbst- lernkurse) werden die Schwerpunkte „Entwicklung von positiven Rahmenbedingungen für den Kindersport“, „,kindund jugendzentriertes Training" sowie das „Planen, Durchführen und Reflektieren von Kindertraining" behandelt. Gerade der erste MOOC nimmt das Selbstverständnis und die Rolle des ${ }^{\star} r$ Trainers*in in den Blick und gibt Möglichkeiten zur Reflexion. Die MOOCs sind frei zugänglich und kostenlos. Ziel des Projekts ist es, allen interessierten Trainer*innen auf der Welt Zugang zur Trainer*inbildung zu verschaffen und ihre pädagogische Handlungskompetenz im Kindertraining zu stärken. Darüber hinaus können auch Lehrteams, ausbildende Sportverbände oder -kreise auf die Materialien zugreifen und sie in ihre Aus- und Fortbildungen integrieren.

Die 10 iCoachKids-Regeln markieren den ganzheitlichen, kindzentrierten Ansatz dieses Selbstlernkurses:

1. Verhalte Dich kindzentriert.

2. Gehe ganzheitlich vor.

3. Beziehe alle Kinder in das Training ein.

4. Sorge für $\mathrm{Spaß}$ und Sicherheit.

5. Werte den Spaß am Sport höher, als die Verbesserung der sportlichen Leistungsfähigkeit.

6. Konzentriere Dich auf grundlegende Fertigkeiten.

7. Binde die Eltern konstruktiv mit ein.

8. Plane das Training so, dass eine Entwicklung der Kinder möglich ist.

9. Verwende unterschiedliche Methoden für ein effektives Lernen.

10. Setze Wettkämpfe unter Entwicklungsgesichtspunkten ein.

Im besten Fall werden auch die Trainer*in-Projekte im Rahmen von „TrainerInSportdeutschland“ am Ende der Projektphase deutlich machen, wie das Selbstverständnis der Trainer*innen unterstützt werden kann. Erste Ergebnisse sind nach der zweiten Evaluierungsphase etwa Anfang des Jahres 2021 zu erwarten. Geplant ist darüber hinaus eine zweite Befragung derselben Trainer*innen und Verbände durchzuführen, um zu prüfen, ob sich im Selbstverständnis der Trainer*innen etwas verändert hat. Die
Trainerakademie Köln des DOSB hat sich im Rahmen von „TrainerInSportdeutschland" explizit dem Selbstverständnis von Trainer*innen gewidmet und wird erste Good-Practice-Beispiele erarbeiten.

Einige Verbände bieten bereits gute Konzepte für die Entwicklung von Trainer*innen, die kindzentriert arbeiten. Die Basketballjugend oder die Judojugend integrieren das Konzept der Persönlichkeits- und Teamentwicklung in ihre Ausbildungen, um Trainer*innen für pädagogische, also entwicklungsfördernde Themen zu sensibilisieren. Es gilt, all diese Akteur ${ }^{\star}$ innen untereinander stärker zu vernetzen sowie bestehende Konzepte und Modelle zur pädagogischen Trainingsqualität bekannter zu machen und in der Sportpraxis stärker zu verbreiten und umzusetzen. Genauso kann die partnerschaftliche Zusammenarbeit zwischen Sportpraxis und Wissenschaft helfen, geeignete Konzepte und Modelle $\mathrm{zu}$ erproben und gezielt in die Aus- und -fortbildungen von Trainer*innen zu integrieren.

\section{Korrespondenzadresse}

\section{Katharina Morlang}

Deutsche Sportjugend

Frankfurt am Main, Deutschland

morlang@dsj.de

Katharina Morlang ist bei der Deutschen Sportjugend Referentin für Bildung und Qualifizierung.

\section{Literatur}

Calmbach, Flaig, Edwards, et al. (2020). SINUSJugendstudie 2020 - Wie ticken Jugendliche? Lebenswelten von Jugendlichen im Alter von 14 bis 17 Jahren in Deutschland. Bonn: bpb. https:// www.bpb.de/shop/buecher/schriftenreihe/ 311857/sinus-jugendstudie-2020-wie-tickenjugendliche.Zugegriffen:30. Okt. 2020.

DOSB https://cdn.dosb.de/alter_Datenbestand/fmdosb/arbeitsfelder/Ausbildung/Rahmenricht linien_2006/Rahmenrichtlinien_fuer_Qualifizie rung_von_2005.pdf.Zugegriffen:30.0kt. 2020.

Geschäftsbereichsübergreifendes Projekt von DOSB/dsj zur Unterstützung von Trainer*innen in Deutschland (2019-2022). https://trainerinsportdeutschland.dosb.de/. Zugegriffen:30.0kt. 2020.

Richartz, H. (2007). Sallen: Kinder im Leistungssport. Chronische Belastungen und protektive Ressourcen. https://www.researchgate.net/ profile/Jeffrey_Sallen/publication/281742068_ Kinder_im_Leistungssport_Chronische Belastungen_und_protektive_Ressourcen/ links/5a0a0363a6fdcc2736dea0b1/Kinder-imLeistungssport-Chronische-Belastungen-und- 
protektive-Ressourcen.pdf.Zugegriffen:30.0kt. 2020.

Schmidt, Neuber, \& Rauschenbach (Hrsg.). (2015a). 3. Deutscher Kinder- und Jugendsportbericht. Kinder- und Jugendsport im Umbruch. Schorndorf: Hofmann.

(2015b). Norwegen: Kinderrechte des Sports. In Schmidt, Neuber \& Rauschenbach (Hrsg.), 3. Deutscher Kinder- und Jugendsportbericht. Kinder- und Jugendsport im Umbruch. Schorndorf: Hofmann.

Sygusch, R. (2007). Psychosoziale Ressourcen im Sport. Ein sportartenorientiertes Förderkonzept für Schule und Verein. Schorndorf: Hofmann.

Sygusch, W. (2005). Persönlichkeits- und Teamentwicklung: Förderung psychosozialer Ressourcen im Gerätturnen. Frankfurt a.M.: Deutscher TurnerBund und Deutsche Sportjugend.

UN-Kinderrechtskonventionhttps://www.kinderrechts konvention.info/un-kinderrechtskonvention-365/. Zugegriffen:30.0kt. 2020. 